\title{
Norm Inequalities for the Fourier Coefficients of Some Almost Periodic Functions
}

\author{
Y. Boryshchak, A. Myers, and Y. Sagher \\ Florida Atlantic University, Department of Mathematical Sciences, Boca Raton, FL
}

May 17, 2019

Keywords and phrases: Finitely additive measure spaces, almost periodic functions, Paley-type theorems

\begin{abstract}
Using C. Fefferman's embedding of a charge space in a measure space allows us to apply standard interpolation theorems to prove norm inequalities for Besicovitch almost periodic functions. This yields an analogue of Paley's Inequality for the Fourier coefficients of periodic functions.
\end{abstract}

\section{Introduction}

The Fourier coefficients of a $2 \pi$-periodic function $f \in L^{1}(-\pi, \pi)$ are $\hat{f}(n):=\frac{1}{2 \pi} \int_{-\pi}^{\pi} e^{-i n x} f(x) d x$ with $n \in \mathbb{Z}$. For $1 \leqslant q \leqslant 2$ and $1 / q+1 / q^{\prime}=1$ the Hausdorff-Young inequality reads (e.g. [10] Chapter XII, Theorem 2.3):

$$
\left(\sum_{n=-\infty}^{\infty}|\hat{f}(n)|^{q^{\prime}}\right)^{1 / q^{\prime}} \leqslant\left(\frac{1}{2 \pi} \int_{-\pi}^{\pi}|f(x)|^{q} d x\right)^{1 / q} .
$$

R. A. E. C. Paley [7] extended the Hausdorff-Young inequality to

$$
\left(\sum_{n=1}^{\infty}\left[n^{1 / q^{\prime}} \hat{f}_{n}^{*}\right]^{q} \frac{1}{n}\right)^{1 / q} \leqslant C(q)\left(\int_{-\pi}^{\pi}|f(x)|^{q} d x\right)^{1 / q}
$$

where $\left\{\hat{f}_{n}^{*}\right\}_{n \in \mathbb{N}}$ is the decreasing rearrangement of the sequence $\{|\hat{f}(n)|\}_{n \in \mathbb{N}}$.

In [1] A. Avantaggiati, G. Bruno and R. Iannacci proved a Hausdorff-Young inequality for almost periodic trigonometric polynomials, i.e., trigonometric polynomials of the form:

$$
P(x)=\sum_{k=1}^{n} a\left(\eta_{k} ; P\right) e^{i \eta_{k} x}, \quad \eta_{k} \in \mathbb{R}, \quad a\left(\eta_{k} ; P\right) \in \mathbb{C}
$$

and for functions that are in the completion of these polynomials - the space of Besicovitch almost periodic functions, $B_{a p}^{q}$, with the norm

$$
\|P\|_{B_{a p}^{q}}=\lim _{\tau \rightarrow \infty}\left(\frac{1}{2 \tau} \int_{-\tau}^{\tau}|P(x)|^{q} d x\right)^{1 / q} .
$$

Since

$$
\left\langle e^{i \eta_{j} x}, e^{i \eta_{k} x}\right\rangle=\lim _{\tau \rightarrow \infty}\left(\frac{1}{2 \tau} \int_{-\tau}^{\tau} e^{i \eta_{j} x} \cdot e^{-i \eta_{k} x} d x\right)= \begin{cases}1 & \eta_{j}=\eta_{k} \\ 0 & \eta_{j} \neq \eta_{k}\end{cases}
$$


the functions $\left\{e^{i \eta_{k} x}\right\}_{k \in \mathbb{N}}$ form an orthonormal system in $B_{a p}^{2}$. For $f \in B_{a p}^{1}$ the Fourier coefficients of $f$ are defined

$$
a(\eta ; f)=\lim _{\tau \rightarrow \infty}\left(\frac{1}{2 \tau} \int_{-\tau}^{\tau} f(x) e^{-i \eta x} d x\right), \quad \eta \in \mathbb{R} .
$$

If $f$ is a periodic function and $\eta \in \mathbb{Z}$ then $a(\eta ; f)=\hat{f}(\eta)$.

In [1] the authors prove the following extension of the Hausdorff -Young inequality:

Theorem 1.1. Let $1 \leqslant q \leqslant 2$ and let $f \in B_{a p}^{q}$. If $\left\{a\left(\eta_{n} ; f\right)\right\}_{n \in \mathbb{N}}$ is a sequence of Fourier coefficients of $f$ then

$$
\left(\sum_{j=1}^{\infty}\left|a\left(\eta_{j} ; f\right)\right|^{q^{\prime}}\right)^{1 / q^{\prime}} \leqslant \limsup _{\tau \rightarrow \infty}\left(\frac{1}{2 \tau} \int_{-\tau}^{\tau}|f(s)|^{q} d s\right)^{1 / q} .
$$

We shall prove a Paley-type theorem for $B_{a p}^{q}$ functions. We denote the Lebesgue measure space on the real line by $(\mathbb{R}, \mathcal{L}(\mathbb{R}), \lambda)$. The definition of the Besicovitch $B_{a p}^{q}$ space as the completion of trigonometric polynomials (3) under the norm $\|\cdot\|_{B_{a p}^{q}}$ suggests that one could look at the set function $\gamma$ on Lebesgue measurable subsets of $\mathbb{R}$ for which the following limit

$$
\gamma(S)=\lim _{\tau \rightarrow \infty} \frac{\lambda(\{S \cap(-\tau, \tau)\})}{2 \tau}
$$

exists. Using Banach limits one extends $\gamma$ to a finitely additive set function on all sets of $\mathcal{L}(\mathbb{R})$. Clearly $\gamma$ is a finitely additive set function, but since

$$
\sum_{n \in \mathbb{Z}} \gamma([n, n+1))=0<1=\gamma\left(\bigcup_{n \in \mathbb{Z}}[n, n+1)\right)
$$

$\gamma$ is not $\sigma$-additive. To handle the lack of $\sigma$-additivity we use C. Fefferman's [4] construction of a measure space $\left(\mathbb{R}^{\prime}, \mathcal{L}^{\prime}\left(\mathbb{R}^{\prime}\right), \gamma^{\prime}\right)$ with respect to which the $L^{q}\left(\mathbb{R}^{\prime}, \mathcal{L}^{\prime}\left(\mathbb{R}^{\prime}\right), \gamma^{\prime}\right)$ norm is equivalent to $\|\cdot\|_{B_{a p}^{q}}$. This enables us to use standard interpolation theorems to prove the following theorem.

Theorem 1.2. Let $1 \leqslant q \leqslant 2$ and let $f \in B_{a p}^{q}$. If $\left\{a\left(\eta_{n} ; f\right)\right\}_{n \in \mathbb{N}}$ is a sequence of Fourier coefficients of $f$ then

$$
\left(\sum_{n=1}^{\infty}\left[n^{1 / q^{\prime}} a^{*}\left(\eta_{n} ; f\right)\right]^{q} \frac{1}{n}\right)^{1 / q} \leqslant C(q)\left(\int_{\mathbb{R}}|f(x)|^{q} d \gamma(x)\right)^{1 / q}
$$

where $\left\{a^{*}\left(\eta_{n} ; f\right)\right\}_{n \in \mathbb{N}}$ is the decreasing rearrangement of the sequence $\left\{\left|a\left(\eta_{n} ; f\right)\right|\right\}_{n \in \mathbb{N}}$.

Since the left hand side of $(10)$ is the $l^{q^{\prime}, q}$-norm of the sequence $\left\{a\left(\eta_{n} ; f\right)\right\}_{n \in \mathbb{N}}$ and for $1<q<2$ the space $l^{q^{\prime}, q}$ is a subspace of $l^{q^{\prime}}$, except for $C(q)$, inequality (10) is stronger than (7). Also, we shall extend (10) to the case $l^{q, r}$ with $0<r<\infty$ and $1<q<2$.

The paper is organized as follows. Section 2 contains a brief theory of finitely additive measures, "charges". From this, some facts on integration and new results on the well-definedness of $L^{p, q}$ for charge spaces, using Cauchy sequences, are presented. In Section 3, we prove that the C. Fefferman construction of a measure space that contains an isometric isomorphic image of a given charge space also preserves $L^{p, q}$ norms. The set function, $\gamma$, in (8) is extended to a charge in Section 4 using Banach Limits. Section 5 combines the results of the previous sections to prove a Paley-type theorem for Besicovitch almost periodic functions. 


\section{Just Enough Theory of Charges}

We present a short outline of part of the theory of charges that is used in the notes and refer the reader to K. Rao and M. Rao [8] for an excellent exposition of the subject. Some proofs of known theorems are not included here, but may be found in [2].

\subsection{Charges, simple functions, and spaces of measurable functions}

Let $\Omega$ be a set and let $\mathcal{F}$ be a collection of subsets of $\Omega$. $\mathcal{F}$ is said to be a field on $\Omega$ if $\Omega \in \mathcal{F}$ and $\mathcal{F}$ is closed under finite unions and set differences. A map $\mu: \mathcal{F} \rightarrow[0, \infty]$ is said to be a charge on $\mathcal{F}$ if $\mu(\varnothing)=0$ and $\mu$ is finitely additive. A probability charge is a charge for which $\mu(\Omega)=1$. The triple $(\Omega, \mathcal{F}, \mu)$ is called a charge space.

For a charge space, $(\Omega, \mathcal{F}, \mu)$, define the outer charge of $E \subseteq \Omega$ by $\boldsymbol{\mu}^{*}(\boldsymbol{E})=\inf \{\mu(F): E \subseteq$ $F, F \in \mathcal{F}\}$. Note that if $E \in \mathcal{F}$, then $\mu^{*}(E)=\mu(E)$. From the finite additivity of $\mu, \mu^{*}$ is finitely, not necessarily $\sigma$, sub-additive and so the Carathéodory charge induced by $\mu^{*}$ is finitely additive [9].

Definition 2.1. Let $(\Omega, \mathcal{F}, \mu)$ be a charge space. A sequence $\left\{f_{n}\right\}$ of real valued functions on $\Omega$ is said to be Cauchy in $\boldsymbol{\mu}^{*}$, if $\forall \delta>0$,

$$
\lim _{m, n \rightarrow \infty} \mu^{*}\left(\left\{w \in \Omega:\left|f_{m}(w)-f_{n}(w)\right|>\delta\right\}\right)=0 .
$$

$\left\{f_{n}\right\}$ is said to converge in $\boldsymbol{\mu}^{*}$ to $f: \Omega \rightarrow \mathbb{R}$ and write $\boldsymbol{f}_{\boldsymbol{n}} \stackrel{\boldsymbol{\mu}^{*}}{\longrightarrow} \boldsymbol{f}$, if $\forall \delta>0$,

$$
\lim _{n \rightarrow \infty} \mu^{*}\left(\left\{w \in \Omega:\left|f(w)-f_{n}(w)\right|>\delta\right\}\right)=0 .
$$

In other words, $f_{n}$ converge to $f$ in outer charge ${ }^{1}$.

Definition 2.2. Let $(\Omega, \mathcal{F}, \mu)$ be a charge space. We say that an extended real valued function $f$ on $\Omega$ is a $\boldsymbol{\mu}$-null function, or just null function, if for every $\varepsilon>0$

$$
\mu^{*}(\{|f|>\varepsilon\})=0
$$

We denote the set of null functions on $(\Omega, \mathcal{F}, \mu)$ by $\mathcal{N}(\Omega, \mathcal{F}, \boldsymbol{\mu})$. We say that two real valued functions $f$ and $g$ on $\Omega$ are equal $\boldsymbol{\mu}$-almost everywhere and write $\boldsymbol{f}=\boldsymbol{g} \boldsymbol{\mu}$-a.e. (or just $f=g$ a.e. ) if $f-g$ is a $\mu$-null function. We say that $\boldsymbol{f} \leqslant \boldsymbol{g} \boldsymbol{\mu}$-a.e. if there exists a null function, $h$, s.t. $f \leqslant g+h$.

Remark 2.3. $f, g \in \mathcal{N}(\Omega, \mathcal{F}, \mu)$ implies that $f+g \in \mathcal{N}(\Omega, \mathcal{F}, \mu)$ and the relation $f=g \mu$-a.e. is an equivalence relation.

Definition 2.4. Let $(\Omega, \mathcal{F}, \mu)$ be a charge space and let $f$ be a real valued function on $\Omega$. Define

$$
\|\boldsymbol{f}\|_{\mathcal{F}(\Omega)}=\min \left(1, \inf _{a>0}\left\{\max \left[a, \mu^{*}(\{|f|>a\})\right]\right\}\right) .
$$

Note that $\|\cdot\|_{\mathcal{F}(\Omega)}$ is a semi-metric, that is to say there are non-zero functions, $f$, such that $\|f\|_{\mathcal{F}(\Omega)}=$ 0 . However, the quotient space of functions modulo the equivalence

$$
f \sim g \quad \Leftrightarrow \quad\|f-g\|_{\mathcal{F}(\Omega)}=0
$$

is a metric space. It is easy to show that convergence in $\|\cdot\|_{\mathcal{F}(\Omega)}$ is convergence in outer charge. Similarly $\left\{f_{n}\right\}$ is Cauchy in $\|\cdot\|_{\mathcal{F}(\Omega)}$ if and only if it is Cauchy in outer charge. Thus we consider the following:

\footnotetext{
${ }^{1}$ Rao and Rao [8] name convergence in $\mu^{*}$, "hazy convergence".
} 
Definition 2.5. We denote the set of equivalence classes of functions on $\Omega$ modulo $\mathcal{N}(\Omega, \mathcal{F}, \mu)$ by $\boldsymbol{F}(\Omega, \mathcal{F}, \boldsymbol{\mu}, \mathbb{R})$ or just by $\boldsymbol{F}(\Omega)$. We denote the equivalence class of $f$ by $[f]$.

Note that $\left(F(\Omega),\|\cdot\|_{\mathcal{F}(\Omega)}\right)$ is a metric space, with

$$
\|[f]\|_{\mathcal{F}(\Omega)}=\|f\|_{\mathcal{F}(\Omega)} .
$$

We will not distinguish between sequences of functions which belong to the same equivalence class. We will also not distinguish between equivalence classes and their representatives.

Definition 2.6. Let $(\Omega, \mathcal{F}, \mu)$ be a charge space. We say that $f$ is a simple function if for some $n \in \mathbb{N}$, disjoint $E_{j} \in \mathcal{F}$ and some $\beta_{j} \in \mathbb{R}, f=\sum_{j=1}^{n} \beta_{j} I_{E_{j}}$. Since $\mathcal{F}$ is closed under finite unions one may assume that $\beta_{j}$ are distinct and write $f$ in the canonical form

$$
f=\sum_{j=1}^{n} \beta_{j} I_{\left\{f=\beta_{j}\right\}} .
$$

Applying the standard completion of a metric space to the space of simple functions on a charge space $(\Omega, \mathcal{F}, \mu)$ we obtain a complete metric space $\dot{T} M(\Omega)$, see [4].

Definition 2.7. We define an equivalence relation on sequences of simple functions that are Cauchy in $\mu^{*}$, and say that $\left\{f_{n}\right\}$ and $\left\{g_{n}\right\}$ belong to the same equivalence class if

$$
\lim _{n \rightarrow \infty}\left\|f_{n}-g_{n}\right\|_{\mathcal{F}(\Omega)}=0 .
$$

We denote the space of all equivalence classes of sequences of simple functions that are Cauchy in $\mu^{*}$ by $\dot{T} M(\Omega, \mathcal{F}, \mu)$.

Definition 2.8. If $\left\{f_{n}\right\} \in \dot{T} M(\Omega)$, we define

$$
\left\|\left\{f_{n}\right\}\right\|_{\dot{T} M(\Omega)}=\lim _{n \rightarrow \infty}\left\|f_{n}\right\|_{\mathcal{F}(\Omega)} .
$$

We denote the null element of $\dot{T} M(\Omega, \mathcal{F}, \mu)$ by $\{0\}$.

Definition 2.9. Let $\left\{f_{n}\right\},\left\{g_{n}\right\} \in \dot{T} M(\Omega, \mathcal{F}, \mu)$. We say that $\left\{f_{n}\right\} \geqslant\{0\}$ if $\left\{f_{n}^{-}\right\}=\{0\}$. If $\left\{f_{n}-g_{n}\right\} \geqslant$ $\{0\}$ we say that $\left\{f_{n}\right\} \geqslant\left\{g_{n}\right\}$.

Definition 2.10. We denote the closure of simple functions in the metric space $\left(F(\Omega),\|\cdot\|_{\mathcal{F}(\Omega)}\right)$ by $\boldsymbol{T} \boldsymbol{M}(\Omega)$. If $f \in T M(\Omega)$ then we say that $f$ is totally measurable, or $T M$-measurable ${ }^{2}$.

Note that $T M(\Omega, \mathcal{F}, \mu)$ is isometrically isomorphic to the subspace of $\dot{T} M(\Omega, \mathcal{F}, \mu)$ of those sequences, $\left\{f_{n}\right\}$, for which there exists a function, $f$, s.t. $f_{n} \stackrel{\mu^{*}}{\longrightarrow} f$.

Remark 2.11. Let $(\Omega, \mathcal{F}, \mu)$ be a charge space and $\left\{f_{n}\right\},\left\{g_{n}\right\} \in \dot{T} M(\Omega)$

1. If $\Psi: \mathbb{R} \rightarrow \mathbb{R}$ is continuous then $\left.\left\{\Psi\left(f_{n}\right)\right\}\right\} \in \dot{T} M(\Omega)$.

2. If $f, g: \Omega \rightarrow \mathbb{R}$ we denote $\boldsymbol{f} \vee \boldsymbol{g}=\max (f, g)$ and $\boldsymbol{f} \wedge \boldsymbol{g}=\min (f, g)$. If $\left\{f_{n}\right\},\left\{g_{n}\right\} \in \dot{T} M(\Omega)$ then $\left\{f_{n} \vee g_{n}\right\} \in \dot{T} M(\Omega)$ and $\left\{f_{n} \wedge g_{n}\right\} \in \dot{T} M(\Omega)$.

3. For any $c_{1}, c_{2} \in \mathbb{R},\left\{c_{1} f_{n}+c_{2} g_{n}\right\} \in \dot{T} M(\Omega)$.

The following example shows that $T M(\Omega)$ may fail to be a complete space, and since $T M(\Omega)$ is a closed subspace of $\mathcal{F}(\Omega)$, the space $\mathcal{F}(\Omega)$ may fail to be complete as well.

Example 2.12. Let $\mathcal{F}$ be the field of finite and co-finite subsets of $\mathbb{N}$. Let $\mu(\{n\})=2^{-n}$ and $\mu(\mathbb{N} \backslash\{n\})=5-2^{-n}$. Let $f_{n}=I_{(0, n]}$. Since $\sum_{n=1}^{\infty} 2^{-n}=1$, it is clear that $\left\{f_{n}\right\}$ is a Cauchy sequence in $\mu^{*}$ and that there is no $f \in T M(\Omega)$ so that $f_{n} \stackrel{\mu^{*}}{\longrightarrow} f$.

\footnotetext{
${ }^{2}$ Rao and Rao [8] refer to these functions as $T_{1}$-measurable
} 


\subsection{Integration on $T M(\Omega)$ and on its completion}

Definition 2.13. Let $(\Omega, \mathcal{F}, \mu)$ be a charge space. We say that a simple function, $f=\sum_{j=1}^{n} \beta_{j} I_{\left\{f=\beta_{j}\right\}}$, is integrable if $\forall \beta_{j} \neq 0 \mu\left(\left\{f=\beta_{j}\right\}\right)<\infty$ and define the integral of $f$ by

$$
\int_{\Omega} f d \mu=\sum_{j=1}^{n} \beta_{j} \mu\left(\left\{f=\beta_{j}\right\}\right) .
$$

The theory of integration in [8] has been developed for $T M(\Omega)$. For our purposes, it is more useful to consider $\dot{T} M(\Omega)$. The fundamental facts of integration remain valid for $\dot{T} M(\Omega)$ and proofs of the following properties for Cauchy sequences, rather than convergent ones, can be modified from the existing proofs or can easily be supplied. In particular, linear combinations of simple integrable functions are integrable, and Chebyshev's inequality for simple functions also holds in charge spaces.

Definition 2.14. Let $(\Omega, \mathcal{F}, \mu)$ be a charge space. If there exists a sequence, $\left\{f_{n}\right\}$, of integrable simple functions on $\Omega$ s.t. $f_{n} \stackrel{\mu^{*}}{\longrightarrow} f$ and

$$
\lim _{m, n \rightarrow \infty} \int\left|f_{n}-f_{m}\right| d \mu=0 .
$$

then $f$ is said to be integrable and

$$
\int f d \mu=\lim _{n \rightarrow \infty} \int f_{n} d \mu
$$

is called the integral of $f$. The sequence $\left\{f_{n}\right\}$ is called a determining sequence for $f$.

If $\left\{f_{n}\right\} \in \dot{T} M(\Omega)$, each $f_{n}$ is integrable and (21) holds, then we say that $\left\{f_{n}\right\}$ is integrable and define

$$
\int_{\Omega}\left\{f_{n}\right\} d \mu=\lim _{n \rightarrow \infty} \int f_{n} d \mu .
$$

Rao and Rao ([8], Proposition 4.4.10) prove that the integral of a $T M$-measurable function is independent of the determining sequence. That the integral of $\left\{f_{n}\right\} \in \dot{T} M(\Omega)$ is independent of the representative from its equivalence class can be proved similarly, or see [2] for an alternative proof.

Definition 2.15. Let $(\Omega, \mathcal{F}, \mu)$ be a charge space and $\left\{f_{n}\right\} \in \dot{T} M(\Omega)$. We say that $\left\{f_{n}\right\}$ is integrable on a set $E \in \mathcal{F}$ if $\left\{f_{n} I_{E}\right\}$ is integrable and define

$$
\int_{E}\left\{f_{n}\right\} d \mu=\lim _{n \rightarrow \infty} \int_{\Omega}\left\{f_{n} I_{E}\right\} d \mu .
$$

Similar to Remark 2.11 for $\dot{T} M(\Omega)$, the integral on $\dot{T} M(\Omega)$ is linear. Also, if $\left\{f_{n}\right\}$ and $\left\{g_{n}\right\}$ are integrable in $\dot{T} M(\Omega)$, then so are $\left\{f_{n} \vee g_{n}\right\}$ and $\left\{f_{n} \wedge g_{n}\right\}$.

\section{3 $\quad L^{p}$ and $L^{p, q}$ spaces of $T M(\Omega)$ and $\dot{T} M(\Omega)$ functions}

Definition $2.16([4])$. Let $(\Omega, \mathcal{F}, \mu)$ be a charge space and $\left\{f_{n}\right\} \in \dot{T} M(\Omega)$. For $0<p<\infty$, we say that $\left\{f_{n}\right\} \in \dot{\boldsymbol{L}}^{p}(\Omega, \mathcal{F}, \boldsymbol{\mu})$ if each $f_{n}$ is integrable and

$$
\lim _{m, n \rightarrow \infty} \int_{\Omega}\left|\left(\left|f_{n}\right|^{p}-\left|f_{m}\right|^{p}\right)\right| d \mu=0 .
$$

Since

$$
\lim _{m, n \rightarrow \infty}\left|\int_{\Omega}\left(\left|f_{n}\right|^{p}-\left|f_{m}\right|^{p}\right) d \mu\right| \leq \lim _{m, n \rightarrow \infty} \int_{\Omega}\left|\left(\left|f_{n}\right|^{p}-\left|f_{m}\right|^{p}\right)\right| d \mu=0 .
$$

$\lim _{n \rightarrow \infty} \int_{\Omega}\left|f_{n}\right|^{p} d \mu$ exists. 
Definition 2.17. If $\left\{f_{n}\right\} \in \dot{L}^{p}(\Omega, \mathcal{F}, \mu)$ we define

$$
\left\|\left\{f_{n}\right\}\right\|_{L^{p}(\Omega)}=\lim _{n \rightarrow \infty}\left\|f_{n}\right\|_{L^{p}(\Omega)} .
$$

Definition 2.18. Let $(\Omega, \mathcal{F}, \mu)$ be a charge space and $\left\{f_{n}\right\} \in \dot{T} M(\Omega)$. We say that $\left\{f_{n}\right\} \in \dot{\boldsymbol{L}}^{\infty}$ if there exists $0<C<\infty$ s.t.

$$
\lim _{n \rightarrow \infty} \mu^{*}\left(\left\{\left|f_{n}\right|>C\right\}\right)=0
$$

If $\left\{f_{n}\right\} \in \dot{L}^{\infty}$ we define

$$
\left\|\left\{f_{n}\right\}\right\|_{L^{\infty}(\Omega)}=\inf \left\{C>0: \lim _{n \rightarrow \infty} \mu^{*}\left(\left\{\left|f_{n}\right|>C\right\}\right)=0\right\} .
$$

Definition 2.19. Define $\boldsymbol{L}^{p}(\Omega, \mathcal{F}, \boldsymbol{\mu})$ to be the space of all $T M$-measurable functions, $f$, s.t. $|f|^{p}$ is integrable and

$$
\|\boldsymbol{f}\|_{L^{p}(\Omega)}=\left(\int_{\Omega}|f|^{p} d \mu\right)^{1 / p}<\infty
$$

We define $\boldsymbol{L}^{\infty}(\Omega, \mathcal{F}, \boldsymbol{\mu})$ to be the space of $T M$-measurable functions that differ from a bounded function by a null function and define the $L^{\infty}$ norm of a $T M$-measurable function, $f$, by

$$
\|\boldsymbol{f}\|_{L^{\infty}(\boldsymbol{\Omega})}=\inf \left\{C>0: \mu^{*}(\{|f|>C\})=0\right\} .
$$

Note that for $0<p \leq \infty, L^{p}(\Omega, \mathcal{F}, \mu)$ is isometrically isomorphic to the subspace of $\dot{L}^{p}(\Omega, \mathcal{F}, \mu)$ of those sequences, $\left\{f_{n}\right\}$, for which there exists a function, $f$, s.t. $f_{n} \stackrel{\mu^{*}}{\longrightarrow} f$.

Definition 2.20. Let $(\Omega, \mathcal{F}, \mu)$ be a charge space and $f: \Omega \rightarrow \mathbb{R}$. For $t>0$ let

$$
\mu_{f}(t)=\mu^{*}(\{|f|>t\})
$$

The function $\mu_{f}: \mathbb{R}^{+} \rightarrow \mathbb{R}^{+}$is called the distribution of $\boldsymbol{f}$ on $(\Omega, \mathcal{F}, \mu)$.

Clearly, $\mu_{f}=\mu_{|f|}$.

Theorem 2.21. Let $f$ and $g$ be simple integrable functions on a charge space $(\Omega, \mathcal{F}, \mu)$. Then:

1. The distribution $\mu_{f}$ is a decreasing integrable function and

$$
\int_{\Omega}|f| d \mu=\int_{0}^{\infty} \mu_{f}(t) d t
$$

2. If $f, g \geqslant 0$ or $f, g \leqslant 0$ then

$$
\int_{\Omega}|f-g| d \mu=\int_{0}^{\infty}\left|\mu_{f \vee g}(t)-\mu_{f \wedge g}(t)\right| d t .
$$

3.

$$
\int_{0}^{\infty}\left|\mu_{f}(t)-\mu_{g}(t)\right| d t \leqslant \int_{\Omega}|(|f|-|g|)| d \mu
$$

Proof.

1. Let $f=\sum_{k=1}^{N} c_{k} I_{\left\{f=c_{k}\right\}}$, with $0=c_{0}<\left|c_{1}\right|<\ldots<\left|c_{N}\right|$. For $k=1, \ldots, N$ let $\alpha_{k}=\left|c_{k}\right|-\left|c_{k-1}\right|$ and $E_{k}=\left\{|f| \geqslant\left|c_{k}\right|\right\}$. Then

$$
|f|=\sum_{k=1}^{N} \alpha_{k} I_{E_{k}} \quad \text { with } \alpha_{k}>0, E_{k+1} \subseteq E_{k}, E_{k} \in \Omega
$$


and so, applying summation by parts,

$$
\int_{\Omega}|f| d \mu=\sum_{k=1}^{N}\left|c_{k}\right| \mu\left(\left\{f=c_{k}\right\}\right)=\sum_{k=1}^{N} \alpha_{k} \mu\left(E_{k}\right)=\int_{0}^{\infty} \mu_{f}(t) d t .
$$

2. It is enough to consider the case $f, g \geqslant 0$. Observe that for any $w \in \Omega, f(w) \wedge g(w)$ is one of $f(w)$ and $g(w)$, and $f(w) \vee g(w)$ is the other. Since $f, g \geqslant 0$, by Part 1 ,

$$
\begin{aligned}
\int_{0}^{\infty}\left|\mu_{f \vee g}(t)-\mu_{f \wedge g}(t)\right| d t & =\int_{0}^{\infty}\left[\mu_{f \vee g}(t)-\mu_{f \wedge g}(t)\right] d t \\
& =\int_{\Omega}(f \vee g-f \wedge g) d \mu \\
& =\int_{\Omega}|f \vee g-f \wedge g| d \mu \\
& =\int_{\Omega}|f-g| d \mu
\end{aligned}
$$

3. Since $|f| \wedge|g| \leqslant|f|,|g| \leqslant|f| \vee|g|$, it follows that $\mu_{|f| \wedge|g|} \leqslant \mu_{|f|}, \mu_{|g|} \leqslant \mu_{|f| \vee|g|}$ and so by part 2 ,

$$
\begin{aligned}
\int_{0}^{\infty}\left|\mu_{f}(t)-\mu_{g}(t)\right| d t & =\int_{0}^{\infty}\left|\mu_{|f|}(t)-\mu_{|g|}(t)\right| d t \\
& \leqslant \int_{0}^{\infty}\left[\mu_{|f| \vee|g|}(t)-\mu_{|f| \wedge|g|}(t)\right] d t=\int_{\Omega}|(|f|-|g|)| d \mu .
\end{aligned}
$$

Corollary 2.22. Let $f$ and $g$ be simple integrable functions on a charge space $(\Omega, \mathcal{F}, \mu)$. Then for any $\delta \geqslant 0$,

$$
\int_{\delta}^{\infty}\left|\mu_{f_{n}}(t)-\mu_{g_{n}}(t)\right| d t \leqslant \int_{\left\{\left|f_{n}\right|>\delta\right\} \cup\left\{\left|g_{n}\right|>\delta\right\}}\left|\left(\left|f_{n}\right|-\left|g_{n}\right|\right)\right| d \mu
$$

Proof.

$$
\begin{aligned}
\int_{\delta}^{\infty}\left|\mu_{f_{n}}(t)-\mu_{g_{n}}(t)\right| d t & =\int_{0}^{\infty}\left|\mu_{f_{n}}(t+\delta)-\mu_{g_{n}}(t+\delta)\right| d t \\
& \leqslant \int_{0}^{\infty}\left|\mu_{\left(\left|f_{n}\right|-\delta\right) \vee 0}(t)-\mu_{\left(\left|g_{n}\right|-\delta\right) \vee 0}(t)\right| d t \\
& \leqslant \int_{\Omega}\left|\left(\left|f_{n}\right|-\delta\right) \vee 0-\left(\left|g_{n}\right|-\delta\right) \vee 0\right| d \mu \\
& =\int_{\Omega}\left|\left(\left|f_{n}\right| \vee \delta\right)-\delta-\left(\left|g_{n}\right| \vee \delta-\delta\right)\right| d \mu \\
& \leqslant \int_{\left\{\left|f_{n}\right|>\delta\right\} \cup\left\{\left|g_{n}\right|>\delta\right\}}\left|\left(\left|f_{n}\right|-\left|g_{n}\right|\right)\right| d \mu
\end{aligned}
$$

We will work with the $L^{p, q}$ space over a charge space. Let us first review this concept on a measure space.

Definition 2.23. Let $(\Omega, \Sigma, \mu)$ be a measure space and let $f: \Omega \rightarrow \mathbb{R}$ be a measurable function. The decreasing rearrangement of $f$, is defined:

$$
\boldsymbol{f}^{*}(\boldsymbol{t})=\inf \left\{\gamma: \mu_{f}(\gamma) \leqslant t\right\}, \quad t>0
$$


Definition 2.24. Let $(\Omega, \Sigma, \mu)$ be a measure space. For $0<p, q<\infty$ and a measurable function $f: \Omega \rightarrow \mathbb{R}$ let

$$
\|f\|_{L^{p, q}(\Omega)}=\left(\int_{0}^{\infty}\left[t^{1 / p} f^{*}(t)\right]^{q} \frac{d t}{t}\right)^{1 / q}
$$

and for $0<p \leqslant \infty$

$$
\|f\|_{L^{p, \infty}(\Omega)}=\lim _{q \rightarrow \infty}\|f\|_{L^{p, q}(\Omega)}=\sup \left[t^{1 / p} f^{*}(t)\right] .
$$

The space of all measurable functions, $f$, such that $\|f\|_{L^{p, q}(\Omega)}<\infty$ is denoted by $\boldsymbol{L}^{p, q}(\Omega, \Sigma, \boldsymbol{\mu})$.

One can also define $\|f\|_{L^{p, q}(\Omega)}$ in terms of $\mu_{f}$ rather than $f^{*}$. This definition is convenient for functions on charge spaces.

Theorem 2.25 ([5], Proposition 1.4.9). Let $(\Omega, \Sigma, \mu)$ be a measure space. If $0<p<\infty, 0<q \leq \infty$ and $f \in L^{p, q}(\Omega, \Sigma, \mu)$ then

$$
\|f\|_{L^{p, q}(\Omega)}=\left(p \int_{0}^{\infty}\left[\mu_{f}(s)\right]^{\frac{q}{p}} S^{q-1} d s\right)^{1 / q}=\left(\frac{p}{q} \int_{0}^{\infty}\left[\mu_{|f| q}(t)\right]^{\frac{q}{p}} d t\right)^{1 / q} .
$$

and

$$
\|f\|_{L^{p, \infty}(\Omega)}=\lim _{q \rightarrow \infty}\|f\|_{L^{p, q}(\Omega)}=\sup _{t>0}\left\{t \mu_{f}^{\frac{1}{p}}(t)\right\}
$$

Motivated by Theorem 2.25 we make the following definitions for functions on charge spaces.

Definition 2.26. Let $f$ be a simple integrable function on a charge space $(\Omega, \mathcal{F}, \mu)$. For $0<p, q<\infty$ let

$$
\|\boldsymbol{f}\|_{L^{p, q}(\Omega)}=\left(p \int_{0}^{\infty}\left[\mu_{f}(s)\right]^{\frac{q}{p}} s^{q-1} d s\right)^{1 / q} .
$$

For $0<p<\infty$,

$$
\|\boldsymbol{f}\|_{L^{p, \infty}(\Omega)}=\sup _{t>0}\left\{t \mu_{f}^{\frac{1}{p}}(t)\right\} .
$$

Since for $q<\infty$,

$$
\mu_{f}(t)=\mu_{|f|^{q}}\left(t^{q}\right),
$$

it follows that for any $0 \leqslant a<b \leqslant \infty$

$$
\int_{a}^{b}\left[\mu_{f}(s)\right]^{\frac{q}{p}} s^{q-1} d s=\frac{1}{q} \int_{a^{q}}^{b^{q}}\left[\mu_{|f|^{q}}(t)\right]^{\frac{q}{p}} d t,
$$

and so one may define $\|f\|_{L^{p, q}(\Omega)}$ in terms of $\mu_{|f|^{q}}$ instead of $\mu_{f}$.

Theorem 2.27. Let $(\Omega, \mathcal{F}, \mu)$ be a charge space and $0<p, q<\infty$. Let $f, g$ be simple integrable functions. Then

$$
\int_{0}^{\infty}\left|\mu_{f}^{q / p}(t)-\mu_{g}^{q / p}(t)\right| t^{q} \frac{d t}{t}=\frac{1}{q} \int_{0}^{\infty}\left|\mu_{|f|^{q}}^{q / p}(t)-\mu_{|g|^{q}}^{q / p}(t)\right| d t .
$$


Proof. There exist $0=a_{1}<a_{2}<\cdots<a_{n-1}<a_{n}=\infty$ so that on each interval $\left(a_{j}, a_{j+1}\right)$ the function $\mu_{f}^{q / p}-\mu_{g}^{q / p}$ does not change signs. Then

$$
\begin{aligned}
\int_{0}^{\infty}\left|\mu_{f}^{q / p}(t)-\mu_{g}^{q / p}(t)\right| t^{q} \frac{d t}{t} & =\sum_{j=1}^{n-1} \int_{a_{j}}^{a_{j+1}}\left|\mu_{f}^{q / p}(t)-\mu_{g}^{q / p}(t)\right| t^{q} \frac{d t}{t} \\
& =\sum_{j=1}^{n-1}\left|\int_{a_{j}}^{a_{j+1}}\left[\mu_{f}^{q / p}(t)-\mu_{g}^{q / p}(t)\right] t^{q} \frac{d t}{t}\right| \\
& =\frac{1}{q} \sum_{j=1}^{n-1}\left|\int_{a_{j}^{q}}^{a_{j+1}^{q}}\left[\mu_{|f|^{q}}^{q / p}(s)-\mu_{|g|^{q}}^{q / p}(s)\right] d s\right| \\
& =\frac{1}{q} \int_{0}^{\infty}\left|\mu_{|f|^{q}}^{q / p}(s)-\mu_{|g|^{q}}^{q / p}(s)\right| d s .
\end{aligned}
$$

Lemma 2.28. Let $(\Omega, \mathcal{F}, \mu)$ be a charge space. Let $\left\{f_{n}\right\},\left\{g_{n}\right\} \in \dot{T} M(\Omega)$ be such that $\forall \varepsilon>0$,

$$
\lim _{n \rightarrow \infty} \mu\left(\left\{\left|f_{n}-g_{n}\right|>\varepsilon\right\}\right)=0 \text {. }
$$

If there exist $\delta \geqslant 0$ and $C_{\delta}, M>0$, such that $\forall n \in \mathbb{N}$,

$$
\left|f_{n}\right| \leqslant M, \quad \mu_{f_{n}}(\delta) \leqslant C_{\delta}, \quad \text { and } \quad\left|g_{n}\right| \leqslant M, \quad \mu_{g_{n}}(\delta) \leqslant C_{\delta}
$$

then for any $0<r<\infty$,

$$
\lim _{n \rightarrow \infty} \int_{\delta}^{\infty}\left|\mu_{f_{n}}^{r}(t)-\mu_{g_{n}}^{r}(t)\right| d t=0
$$

Proof. By (54),

$$
\int_{\delta}^{\infty}\left|\mu_{f_{n}}^{r}(t)\right| d t \leqslant C_{\delta}^{r} M \quad \text { and } \quad \int_{\delta}^{\infty}\left|\mu_{g_{n}}^{r}(t)\right| d t \leqslant C_{\delta}^{r} M .
$$

For $\varepsilon>0$ let $A_{n}^{\varepsilon}=\left\{\left|\mu_{f_{n}}^{r}-\mu_{g_{n}}^{r}\right|>\varepsilon\right\}$. Then

$$
\begin{aligned}
\int_{\delta}^{\infty}\left|\mu_{f_{n}}^{r}(t)-\mu_{g_{n}}^{r}(t)\right| d t & =\int_{\delta}^{M}\left|\mu_{f_{n}}^{r}(t)-\mu_{g_{n}}^{r}(t)\right| d t \\
& =\int_{[\delta, M] \cap A_{n}^{\varepsilon}}\left|\mu_{f_{n}}^{r}(t)-\mu_{g_{n}}^{r}(t)\right| d t+\int_{[\delta, M] \cap\left(A_{n}^{\varepsilon}\right)^{c}}\left|\mu_{f_{n}}^{r}(t)-\mu_{g_{n}}^{r}(t)\right| d t \\
& \leqslant \int_{[\delta, M] \cap A_{n}^{\varepsilon}}\left|\mu_{f_{n}}^{r}(t)-\mu_{g_{n}}^{r}(t)\right| d t+\varepsilon M .
\end{aligned}
$$

Let us see that

$$
\lim _{n \rightarrow \infty} \int_{[\delta, M] \cap A_{n}^{\varepsilon}}\left|\mu_{f_{n}}^{r}(t)-\mu_{g_{n}}^{r}(t)\right| d t=0 .
$$

For any $\varepsilon>0$ there exists $0<\alpha<\varepsilon$ so that $|x-y| \leqslant \alpha$ implies $\left|x^{r}-y^{r}\right|<\varepsilon$. If $t \in A_{n}^{\varepsilon}$ then $\left|\mu_{f_{n}}^{r}(t)-\mu_{g_{n}}^{r}(t)\right|>\varepsilon$ and therefore $\left|\mu_{f_{n}}(t)-\mu_{g_{n}}(t)\right|>\alpha$.

By (53), for any $\varepsilon_{1}>0$ there exists $N \in \mathbb{N}$ so that for all $n \geqslant N$

$$
\begin{aligned}
\int_{\left\{\left|f_{n}\right|>\delta\right\} \cup\left\{\left|g_{n}\right|>\delta\right\}}\left|\left(\left|f_{n}\right|-\left|g_{n}\right|\right)\right| d \mu & =\int_{\left\{\left|f_{n}-g_{n}\right|>\varepsilon_{1}\right\} \cap\left(\left\{\left|f_{n}\right|>\delta\right\} \cup\left\{\left|g_{n}\right|>\delta\right\}\right)}\left|\left(\left|f_{n}\right|-\left|g_{n}\right|\right)\right| d \mu \\
& +\int_{\left\{\left|f_{n}-g_{n}\right| \leqslant \varepsilon_{1}\right\} \cap\left(\left\{\left|f_{n}\right|>\delta\right\} \cup\left\{\left|g_{n}\right|>\delta\right\}\right)}\left|\left(\left|f_{n}\right|-\left|g_{n}\right|\right)\right| d \mu \\
& \leqslant M \varepsilon_{1}+2 C_{\delta} \varepsilon_{1} .
\end{aligned}
$$


It follows that

$$
\lim _{n \rightarrow \infty} \int_{\left\{\left|f_{n}\right|>\delta\right\} \cup\left\{\left|g_{n}\right|>\delta\right\}}\left|\left(\left|f_{n}\right|-\left|g_{n}\right|\right)\right| d \mu=0 .
$$

By Chebyshev's inequality and Corollary 2.22, for any $\alpha>0$,

$$
\alpha \lambda\left(\left\{t>\delta:\left|\mu_{f_{n}}(t)-\mu_{g_{n}}(t)\right|>\alpha\right\}\right) \leqslant \int_{\delta}^{\infty}\left|\mu_{f_{n}}(t)-\mu_{g_{n}}(t)\right| d t \leqslant \int_{\left\{\left|f_{n}\right|>\delta\right\} \cup\left\{\left|g_{n}\right|>\delta\right\}}\left|\left(\left|f_{n}\right|-\left|g_{n}\right|\right)\right| d \mu
$$

proving

$$
\lim _{n \rightarrow \infty} \lambda\left(A_{n}^{\varepsilon} \cap(\delta, \infty)\right)=0
$$

and by (54)

$$
\lim _{n \rightarrow \infty} \int_{[\delta, M] \cap A_{n}^{\varepsilon}}\left|\mu_{g_{n}}^{r}(t)-\mu_{f_{n}}^{r}(t)\right| d t \leqslant \lim _{n \rightarrow \infty} C_{\delta}^{r} \cdot \lambda\left(A_{n}^{\varepsilon}\right)=0
$$

Theorem 2.29. Let $(\Omega, \mathcal{F}, \mu)$ be a charge space and $0<r<\infty$. Let $\left\{f_{n}\right\},\left\{g_{n}\right\} \in \dot{T} M(\Omega, \mathcal{F}, \mu)$ be sequences of simple integrable functions so that (53) holds. If also

$$
\lim _{m, n \rightarrow \infty} \int_{0}^{\infty}\left|\mu_{f_{n}}^{r}(t)-\mu_{f_{m}}^{r}(t)\right| d t=0, \quad \lim _{m, n \rightarrow \infty} \int_{0}^{\infty}\left|\mu_{g_{n}}^{r}(t)-\mu_{g_{m}}^{r}(t)\right| d t=0,
$$

then

$$
\lim _{n \rightarrow \infty} \int_{0}^{\infty} \mu_{f_{n}}^{r}(t) d t=\lim _{n \rightarrow \infty} \int_{0}^{\infty} \mu_{g_{n}}^{r}(t) d t
$$

Proof. Since

$$
\left|\int_{0}^{\infty} \mu_{f_{n}}^{r}(t) d t-\int_{0}^{\infty} \mu_{g_{n}}^{r}(t) d t\right| \leqslant \int_{0}^{\infty}\left|\mu_{f_{n}}^{r}(t)-\mu_{g_{n}}^{r}(t)\right| d t
$$

if (65) fails, then there exist $\varepsilon>0$ and subsequences $\left\{f_{n_{k}}\right\}$ and $\left\{g_{n_{k}}\right\}$ that satisfy (53), so that for all $k \in \mathbb{N}$,

$$
\int_{0}^{\infty}\left|\mu_{f_{n_{k}}}^{r}(t)-\mu_{g_{n_{k}}}^{r}(t)\right| d t>\varepsilon
$$

To simplify the notation, we will use $\left\{f_{n}\right\}$ and $\left\{g_{n}\right\}$ instead of $\left\{f_{n_{k}}\right\}$ and $\left\{g_{n_{k}}\right\}$. By (64), there exists $N_{1} \in \mathbb{N}$ such that for all $n, m \geqslant N_{1}$,

$$
\int_{0}^{\infty}\left|\mu_{f_{n}}^{r}(t)-\mu_{f_{m}}^{r}(t)\right| d t+\int_{0}^{\infty}\left|\mu_{g_{m}}^{r}(t)-\mu_{g_{n}}^{r}(t)\right| d t<\varepsilon / 12
$$

Let $M=\max \left\{\max \left(\left|f_{N_{1}}\right|\right), \max \left(\left|g_{N_{1}}\right|\right)\right\}$ and $S=\max \left\{\mu\left(\operatorname{supp}\left(f_{N_{1}}\right)\right), \mu\left(\operatorname{supp}\left(g_{N_{1}}\right)\right)\right\}$.

Since $f_{N_{1}}$ and $g_{N_{1}}$ are simple integrable functions, $M, S<\infty$ and for all $t>M, \mu_{f_{N_{1}}}(t)=\mu_{g_{N_{1}}}(t)=$ 0. By (68), for all $n>N_{1}$,

$$
\begin{aligned}
\int_{M}^{\infty}\left|\mu_{f_{n}}^{r}(t)-\mu_{g_{n}}^{r}(t)\right| d t & \leqslant \int_{M}^{\infty}\left|\mu_{f_{n}}^{r}(t)\right| d t+\int_{M}^{\infty}\left|\mu_{g_{n}}^{r}(t)\right| d t \\
& =\int_{M}^{\infty}\left|\mu_{f_{n}}^{r}(t)-\mu_{f_{N_{1}}}^{r}(t)\right| d t+\int_{M}^{\infty}\left|\mu_{g_{n}}^{r}(t)-\mu_{g_{N_{1}}}^{r}(t)\right| d t \\
& \leqslant \int_{0}^{\infty}\left|\mu_{f_{n}}^{r}(t)-\mu_{f_{N_{1}}}^{r}(t)\right| d t+\int_{0}^{\infty}\left|\mu_{g_{n}}^{r}(t)-\mu_{g_{N_{1}}}^{r}(t)\right| d t \\
& <\varepsilon / 12 .
\end{aligned}
$$


Since $\left|f_{N_{1}}\right|$ and $\left|g_{N_{1}}\right|$ are integrable functions there exist $\delta>0$ s.t.

$$
\int_{0}^{\delta} \mu_{f_{N_{1}}}^{r}(t) d t<\varepsilon / 12 \text { and } \int_{0}^{\delta} \mu_{g_{N_{1}}}^{r}(t) d t<\varepsilon / 12
$$

By $(64), \forall n>N_{1}$

$$
\begin{aligned}
& \int_{0}^{\delta} \mu_{f_{n}}^{r}(t) d t \leqslant \int_{0}^{\delta} \mu_{f_{N_{1}}}^{r}(t) d t+\int_{0}^{\delta}\left|\mu_{f_{n}}^{r}-\mu_{f_{N_{1}}}^{r}(t)\right| d t<\varepsilon / 6 \\
& \int_{0}^{\delta} \mu_{g_{n}}^{r}(t) d t \leqslant \int_{0}^{\delta} \mu_{g_{N_{1}}}^{r}(t) d t+\int_{0}^{\delta}\left|\mu_{g_{n}}^{r}-\mu_{g_{N_{1}}}^{r}(t)\right| d t<\varepsilon / 6
\end{aligned}
$$

and therefore

$$
\int_{0}^{\delta}\left|\mu_{f_{n}}^{r}(t)-\mu_{g_{n}}^{r}(t)\right| d t<\varepsilon / 3
$$

Let us see that

$$
\lim _{n \rightarrow \infty} \int_{\delta}^{M}\left|\mu_{f_{n}}^{r}(t)-\mu_{g_{n}}^{r}(t)\right| d t=0 .
$$

Since $\mu_{f_{n}}^{r}(t)$ and $\mu_{g_{n}}^{r}(t)$ are decreasing functions, by Chebyshev's inequality and (71), (72), $\forall n>N_{1}$,

$$
\delta \mu_{f_{n}}^{r}(\delta) \leqslant \int_{0}^{\delta} \mu_{f_{n}}^{r}(t) d t<\varepsilon / 6, \quad \delta \mu_{g_{n}}^{r}(\delta) \leqslant \int_{0}^{\delta} \mu_{g_{n}}^{r}(t) d t<\varepsilon / 6,
$$

and so for $C_{\delta}=\left(\frac{\varepsilon}{6 \delta}\right)^{1 / r}$,

$$
\sup _{n \in \mathbb{N}} \mu_{f_{n}}(\delta) \leqslant C_{\delta} \quad \text { and } \quad \sup _{n \in \mathbb{N}} \mu_{g_{n}}(\delta) \leqslant C_{\delta}
$$

Let

$$
\tilde{f}_{n}(x)=\left|f_{n}\right| \wedge M \quad \text { and } \quad \tilde{g}_{n}(x)=\left|g_{n}\right| \wedge M .
$$

Observe that for all $n \in \mathbb{N}$ and all $\delta \leqslant t<M$,

$$
\mu_{f_{n}}(t)=\mu_{\tilde{f}_{n}}(t) \quad \text { and } \quad \mu_{g_{n}}(t)=\mu_{\tilde{g}_{n}}(t) .
$$

Since both $\left|\tilde{f}_{n}\right| \leqslant M,\left|\tilde{g}_{n}\right| \leqslant M$, and by $(76) \mu_{\tilde{f}_{n}}(\delta) \leqslant C_{\delta}, \mu_{\tilde{g}_{n}}(\delta) \leqslant C_{\delta}$ and the conditions (53) hold for $\left\{\tilde{f}_{n}\right\}$ and $\left\{\tilde{g}_{n}\right\}$. By Lemma 2.28 and (78),

$$
\lim _{n \rightarrow \infty} \int_{\delta}^{M}\left|\mu_{f_{n}}^{r}(t)-\mu_{g_{n}}^{r}(t)\right| d t=\lim _{n \rightarrow \infty} \int_{\delta}^{M}\left|\mu_{\tilde{f}_{n}}^{r}(t)-\mu_{\tilde{g}_{n}}^{r}(t)\right| d t=0
$$

and so there exists $N>N_{1}$ such that for all $n, m \geqslant N$,

$$
\int_{\delta}^{M}\left|\mu_{f_{n}}^{r}(t)-\mu_{g_{n}}^{r}(t)\right| d t<\varepsilon / 3
$$

and so (80), (73) and (69) contradict (67).

Definition 2.30. Let $(\Omega, \mathcal{F}, \mu)$ be a charge space and $0<p, q<\infty$. We say that $\left\{f_{n}\right\} \in \dot{L}^{p, q}(\Omega, \mathcal{F}, \mu)$ if $\left\{f_{n}\right\} \in \dot{T} M(\Omega)$ is a sequence of simple integrable functions such that

$$
\lim _{m, n \rightarrow \infty} \int_{0}^{\infty}\left|\mu_{f_{n}}^{q / p}(t)-\mu_{f_{m}}^{q / p}(t)\right| t^{q} \frac{d t}{t}=0
$$

We define

$$
\left\|\left\{\boldsymbol{f}_{\boldsymbol{n}}\right\}\right\|_{\dot{\boldsymbol{L}}^{p, q}(\boldsymbol{\Omega})}=\lim _{n \rightarrow \infty}\left(p \int_{0}^{\infty} \mu_{f_{n}}^{q / p}(t) t^{q} \frac{d t}{t}\right)^{1 / q}
$$


From Theorems 2.27 and 2.29 , it follows that if $\left\{f_{n}\right\}$ and $\left\{g_{n}\right\}$ are equivalent in $\dot{T} M(\Omega)$ and each is in $\dot{L}^{p, q}$, then $\left\|\left\{f_{n}\right\}\right\|_{\dot{L}^{p, q}(\Omega)}=\left\|\left\{g_{n}\right\}\right\|_{\dot{L}^{p, q}(\Omega)}$.

Remark 2.31. Equivalence of representatives in $\dot{T} M(\Omega)$ does not necessarily imply equivalence in $\dot{L}^{p, q}(\Omega)$. For example, the sequence $\left\{n^{2 / p} I_{\left[0, n^{-1}\right]}\right\}$ is a representative of $\{0\}$ in $\dot{T} M(\Omega)$, but is not a representative of $\{0\}$ in $\dot{L}^{p, q}(\Omega)$ since $\|\{0\}\|_{\dot{L}^{p, q}(\mathbb{R})}=0$, but $\lim _{n \rightarrow \infty}\left\|f_{n}\right\|_{L^{p, q}(\mathbb{R})}=\infty$.

Theorem 2.32. Let $(\Omega, \mathcal{F}, \mu)$ be a charge space and $0<p<\infty$. Let $\left\{f_{n}\right\},\left\{g_{n}\right\} \in \dot{T} M(\Omega)$ be sequences of simple integrable functions such that $\forall \varepsilon>0$,

$$
\lim _{n \rightarrow \infty} \mu\left(\left\{\left|f_{n}-g_{n}\right|>\varepsilon\right\}\right)=0
$$

If

$$
\lim _{n, m \rightarrow \infty}\left(\sup _{t>0}\left\{t^{p}\left|\mu_{f_{n}}(t)-\mu_{f_{m}}(t)\right|\right\}\right)=\lim _{n, m \rightarrow \infty}\left(\sup _{t>0}\left\{t^{p}\left|\mu_{g_{n}}(t)-\mu_{g_{m}}(t)\right|\right\}\right)=0
$$

then

$$
\lim _{n \rightarrow \infty} \sup _{t>0}\left\{t^{p} \mu_{f_{n}}(t)\right\}=\lim _{n \rightarrow \infty} \sup _{t>0}\left\{t^{p} \mu_{g_{n}}(t)\right\}
$$

Proof. Let $\varepsilon>0$. Taking subsequences, we may assume

$$
\mu_{f_{n}-g_{n}}(1 / n)=\mu\left(\left\{\left|f_{n}-g_{n}\right|>\frac{1}{n}\right\}\right)<\frac{1}{n}, \quad \forall n \in \mathbb{N} .
$$

By (84), there exists $N_{1} \in \mathbb{N}$ so that $\forall n \geq N_{1}$,

$$
\sup _{t>0}\left\{t^{p}\left|\mu_{f_{n}}(t)-\mu_{f_{N_{1}}}(t)\right|\right\}<\varepsilon / 9 .
$$

There also exists $t_{N_{1}}>0$ so that

$$
\sup _{t>0}\left\{t^{p} \mu_{f_{N_{1}}}(t)\right\} \leq t_{N_{1}}^{p} \mu_{f_{N_{1}}}\left(t_{N_{1}}\right)+\varepsilon / 9 .
$$

For any $n \geq N_{1}$,

$$
\begin{aligned}
t_{N_{1}}^{p} \mu_{f_{n}}\left(t_{N_{1}}\right) & \leq \sup _{t>0}\left\{t^{p} \mu_{f_{n}}(t)\right\} \leq \sup _{t>0}\left\{t^{p}\left|\mu_{f_{n}}(t)-\mu_{f_{N_{1}}}(t)\right|\right\}+\sup _{t>0}\left\{t^{p} \mu_{f_{N_{1}}}(t)\right\} \\
& <2 \varepsilon / 9+t_{N_{1}}^{p} \mu_{f_{N_{1}}}\left(t_{N_{1}}\right)
\end{aligned}
$$

which implies $\mu_{f_{n}}\left(t_{N_{1}}\right) \leq \varepsilon / t_{N_{1}}^{p}+\mu_{f_{N_{1}}}\left(t_{N_{1}}\right)$, and

$$
\begin{aligned}
\sup _{t>0}\left\{t^{p} \mu_{f_{n}}(t)\right\} & \leq 2 \varepsilon / 9+t_{N_{1}}^{p} \mu_{f_{N_{1}}}\left(t_{N_{1}}\right)-t_{N_{1}}^{p} \mu_{f_{n}}\left(t_{N_{1}}\right)+t_{N_{1}}^{p} \mu_{f_{n}}\left(t_{N_{1}}\right) \\
& \leq 2 \varepsilon / 9+t_{N_{1}}^{p}\left|\mu_{f_{n}}\left(t_{N_{1}}\right)-\mu_{f_{N_{1}}}\left(t_{N_{1}}\right)\right|+t_{N_{1}}^{p} \mu_{f_{n}}\left(t_{N_{1}}\right) \\
& \leq 2 \varepsilon / 9+\sup _{t>0}\left\{t^{p}\left|\mu_{f_{n}}(t)-\mu_{f_{N_{1}}}(t)\right|\right\}+t_{N_{1}}^{p} \mu_{f_{n}}\left(t_{N_{1}}\right) \\
& \leq \varepsilon / 3+t_{N_{1}}^{p} \mu_{f_{n}}\left(t_{N_{1}}\right) .
\end{aligned}
$$

Similarly, there exists $N_{2} \in \mathbb{N}$ and $t_{N_{2}}>0$ so that $\forall n \geq N_{2}$,

$$
\sup _{t>0}\left\{t^{p} \mu_{g_{n}}(t)\right\} \leq t_{N_{2}}^{p} \mu_{g_{n}}\left(t_{N_{2}}\right)+\varepsilon / 3 \quad \text { and } \quad \mu_{g_{n}}\left(t_{N_{2}}\right) \leq \varepsilon / t_{N_{2}}^{p}+\mu_{g_{N_{2}}}\left(t_{N_{2}}\right) .
$$

Set $A=\max \left(\varepsilon / t_{N_{1}}^{p}+\mu_{f_{N_{1}}}\left(t_{N_{1}}\right), \varepsilon / t_{N_{2}}^{p}+\mu_{g_{N_{2}}}\left(t_{N_{2}}\right)\right)$. Let $N>\max \left(N_{1}, N_{2}, 3 t_{N_{1}}^{p} / \varepsilon, 3 t_{N_{2}}^{p} / \varepsilon\right)$ and

$$
\max \left(\left|t_{N_{1}}^{p}-\left(t_{N_{1}}-1 / N\right)^{p}\right|,\left|t_{N_{2}}^{p}-\left(t_{N_{2}}-1 / N\right)^{p}\right|\right)<\frac{\varepsilon}{3+A}
$$


Let $n>N$ and without loss of generality, assume $\sup _{t>0}\left\{t^{p} \mu_{f_{n}}(t)\right\} \geq \sup _{t>0}\left\{t^{p} \mu_{g_{n}}(t)\right\}$. Noting that for $0<\delta<t$,

$$
\mu_{f_{n}}(t) \leq \mu_{f_{n}-g_{n}}(\delta)+\mu_{g_{n}}(t-\delta)
$$

it follows

$$
\begin{aligned}
\left|\sup _{t>0}\left\{t^{p} \mu_{f_{n}}(t)\right\}-\sup _{t>0}\left\{t^{p} \mu_{g_{n}}(t)\right\}\right| & \leq t_{N_{1}}^{p} \mu_{f_{n}}\left(t_{N_{1}}\right)+\varepsilon / 3-\left(t_{N_{1}}-1 / N\right)^{p} \mu_{g_{n}}\left(t_{N_{1}}-1 / N\right) \\
& \leq t_{N_{1}}^{p} \mu_{f_{n}}\left(t_{N_{1}}\right)+\varepsilon / 3-\left(t_{N_{1}}-1 / N\right)^{p}\left[\mu_{f_{n}}\left(t_{N_{1}}\right)-\mu_{f_{n}-g_{n}}(1 / N)\right] \\
& \leq \mu_{f_{n}}\left(t_{N_{1}}\right)\left|t_{N_{1}}^{p}-\left(t_{N_{1}}-1 / N\right)^{p}\right|+\varepsilon / 3+\varepsilon / 3<\varepsilon .
\end{aligned}
$$

Having obtained the conclusion for subsequences of $\left\{f_{n}\right\}$ and $\left\{g_{n}\right\}$, the conclusion follows as the limits in (85) exist.

Definition 2.33. Let $(\Omega, \mathcal{F}, \mu)$ be a charge space and $0<p<\infty$. We say that $\left\{f_{n}\right\} \in \dot{T} M(\Omega)$ belongs to the space $\dot{\boldsymbol{L}}^{p, \infty}(\Omega, \mathcal{F}, \boldsymbol{\mu})$ if each $f_{n}$ is integrable and

$$
\lim _{m, n \rightarrow \infty}\left(\sup _{t>0}\left\{t\left|\mu_{f_{n}}^{\frac{1}{p}}(t)-\mu_{f_{m}}^{\frac{1}{p}}(t)\right|\right\}\right)=0 .
$$

We define

$$
\left\|\left\{\boldsymbol{f}_{n}\right\}\right\|_{\dot{\boldsymbol{L}}^{p, \infty}(\boldsymbol{\Omega})}=\lim _{n \rightarrow \infty}\left\|f_{n}\right\|_{L^{p, \infty}(\Omega)}=\lim _{n \rightarrow \infty} \sup _{t>0}\left\{t \mu_{f_{n}}^{\frac{1}{p}}(t)\right\}
$$

\section{Embedding of a charge space into a measure space}

\subsection{A Theorem of C. Fefferman}

In [4] C. Fefferman embedded charge spaces in measure spaces. His result is summarized in parts (1) - (3) of Theorem 3.1. The application to $L^{p, q}$ spaces necessitates a modest extension and this is part (4) of Theorem 3.1.

The notation we use in this chapter is consistent with the notation of the previous two chapters but is different from the notation used in [4].

Theorem 3.1. Let $(\Omega, \mathcal{F}, \mu)$ be a charge space. There is a measure space $\left(\Omega^{\prime}, \Sigma^{\prime}, \mu^{\prime}\right)$ and an orderpreserving, multiplication-preserving isometric isomorphism $\phi$ from $\dot{T} M(\Omega, \mathcal{F}, \mu)$ onto $T M\left(\Omega^{\prime}, \Sigma^{\prime}, \mu^{\prime}\right)$, which we denote by $\mathcal{M}\left(\Omega^{\prime}, \Sigma^{\prime}, \mu^{\prime}\right)$, such that the following holds:

1. If $\left\{f_{n}\right\} \in \dot{T} M(\Omega, \mathcal{F}, \mu)$ is an indicator function, then $\phi\left(\left\{f_{n}\right\}\right) \in \mathcal{M}\left(\Omega^{\prime}, \Sigma^{\prime}, \mu^{\prime}\right)$ is an indicator function. If $\left\{f_{n}\right\} \in \dot{T} M(\Omega, \mathcal{F}, \mu)$ is a simple function, then $\phi\left(\left\{f_{n}\right\}\right) \in \mathcal{M}\left(\Omega^{\prime}, \Sigma^{\prime}, \mu^{\prime}\right)$ is a simple function.

2. If $1 \leqslant p \leqslant \infty$, then $\phi$ takes $\dot{L}^{p}(\Omega, \mathcal{F}, \mu)$ onto $L^{p}\left(\Omega^{\prime}, \Sigma^{\prime}, \mu^{\prime}\right)$ preserving the $L^{p}$ norm.

3. If $\left\{f_{n}\right\} \in \dot{L}^{1}(\Omega, \mathcal{F}, \mu)$, then $\int_{\Omega}\left\{f_{n}\right\} d \mu=\int_{\Omega^{\prime}} \phi\left(\left\{f_{n}\right\}\right) d \mu^{\prime}$.

4. If $0<p, q<\infty$, then $\phi$ takes $\dot{L}^{p, q}(\Omega, \mathcal{F}, \mu)$ onto $L^{p, q}\left(\Omega^{\prime}, \Sigma^{\prime}, \mu^{\prime}\right)$ preserving the $L^{p, q}$ norm. It also maps $\dot{L}^{p, \infty}(\Omega, \mathcal{F}, \mu)$ onto $\dot{L}^{p, \infty}\left(\Omega^{\prime}, \Sigma^{\prime}, \mu^{\prime}\right)$ preserving the $L^{p, \infty}$ norm.

We include the following background material from [4] for the convenience of the reader.

Definition 3.2. Let $\mathcal{B}_{0}$ be the subset of $\dot{T} M(\Omega, \mathcal{F}, \mu)$ of all indicator functions of sets in the field $\mathcal{F}$. Let $\mathcal{B}$ be the closure of $\mathcal{B}_{0}$ in $\dot{T} M(\Omega, \mathcal{F}, \mu)$ with respect to the metric defined in (18). 
Definition 3.3. We define the union function $\bigcup_{0}: \mathcal{B}_{0} \times \mathcal{B}_{0} \rightarrow \mathcal{B}_{0} \subseteq \mathcal{B}$, by

$$
\bigcup_{0}\left(I_{E}, I_{F}\right)=I_{E \cup F}
$$

Since $\bigcup_{0}$ is uniformly continuous on $\mathcal{B}_{0} \times \mathcal{B}_{0}$, we denote the uniformly continuous extension of $\bigcup_{0}$ to a function $\bigcup: \mathcal{B} \times \mathcal{B} \rightarrow \mathcal{B}$.

Definition 3.4. The function $N_{0}: \mathcal{B}_{0} \rightarrow \mathcal{B}_{0} \subseteq \mathcal{B}$ is defined by

$$
N_{0}\left(I_{E}\right)=I_{\Omega \backslash E} .
$$

Since $\left|I_{E}-I_{F}\right|=\left|I_{(\Omega \backslash E)}-I_{(\Omega \backslash F)}\right|, N_{0}$ is an isometry and so extends to a uniformly continuous function $N: \mathcal{B} \rightarrow \mathcal{B}$. Consistent with the notation of Boolean algebras, we denote $N(F)$ by $\sim F$.

Definition 3.5. Define the intersection on $\mathcal{B} \times \mathcal{B}$ by

$$
\left\{I_{F_{n}}\right\} \cap\left\{I_{G_{n}}\right\}=\left\{I_{F_{n} \cap G_{n}}\right\} \quad\left\{I_{F_{n}}\right\},\left\{I_{G_{n}}\right\} \in \mathcal{B} .
$$

Since $\cap$ is a composition of the complementations and unions which are uniformly continuous functions, it is also uniformly continuous.

We will now define a charge on $\mathcal{B}$. If $\left\{I_{E_{n}}\right\}$ and $\left\{I_{F_{n}}\right\}$ are Cauchy in charge and $\left\{I_{E_{n}}\right\}=\left\{I_{F_{n}}\right\}$, that is,

$$
\lim _{n \rightarrow \infty}\left\|I_{E_{n}}-I_{F_{n}}\right\|_{\mathcal{F}(\Omega)}=0
$$

it follows that

$$
\lim _{n \rightarrow \infty} \mu\left(E_{n}\right)=\lim _{n \rightarrow \infty} \mu\left(F_{n}\right)
$$

which justifies:

Definition 3.6. For $E=\left\{I_{E_{n}}\right\} \in \mathcal{B}$, let

$$
\boldsymbol{\mu}_{1}(\boldsymbol{E})=\lim _{n \rightarrow \infty} \mu\left(E_{n}\right)
$$

Lemma 3.7. ${ }^{3}(\mathcal{B}, \cap, \cup, \sim)$ is a Boolean algebra and $\mu_{1}$ is positive and finitely additive on $\mathcal{B}$. If $F \in \mathcal{B}$ and $\mu_{1}(F)=0$, then $F$ is the null element of the Boolean algebra.

By the Stone Representation Theorem ([3]), there is a compact extremally disconnected space, $\Omega^{\prime}$, and a field $\mathcal{F}^{\prime}$ of subsets of $\Omega^{\prime}$ so that $\mathcal{F}^{\prime}$ is isomorphic as a Boolean algebra with $\mathcal{B}$. The set of subsets which are both closed and open is closed under finite unions intersections and complements and therefore forms a field. Let $\phi_{1}: \mathcal{B} \rightarrow \mathcal{F}^{\prime}$ denote the isomorphism. Then $\phi_{1}$ induces a positive, finitely additive set function $\mu_{1}^{\prime}$ on $\mathcal{F}^{\prime}$ defined in the obvious way using $\phi_{1}$ and $\mu_{1}$. The isomorphism proves that the conclusion of Lemma 3.7 holds on $\left(\Omega^{\prime}, \mathcal{F}^{\prime}, \mu_{1}^{\prime}\right)$. The field $\mathcal{F}^{\prime}$ need not be a sigma-field, however,

Lemma 3.8. $\mu_{1}^{\prime}$ is countably additive on $\mathcal{F}^{\prime}$.

Proof. Let $A_{1}, A_{2}, \ldots \in \mathcal{F}^{\prime}$ be disjoint and let $A=\bigcup_{i=1}^{\infty} A_{i}$. If $A \in \mathcal{F}^{\prime}$ then $A$ is closed, hence compact. Since each $A_{i} \in \mathcal{F}^{\prime}$ is open, $\left\{A_{i}\right\}_{i=1}^{\infty}$ is an open cover of $A$, and it follows that only finitely many of the $A_{i} \neq \varnothing$, proving that $\mu_{1}^{\prime}$ is (trivially) countably additive on $\mathcal{F}^{\prime}$.

\footnotetext{
${ }^{3}$ Lemma 1 in [4].
} 
Since $\mu_{1}^{\prime}$ is countably additive on $\mathcal{F}^{\prime}$, by Carathéodory's Theorem there exists an extension of $\mu_{1}^{\prime}$ to a positive measure $\mu^{\prime}$ on $\Sigma^{\prime}$, the sigma field generated by $\mathcal{F}^{\prime}$. We shall show that $\left(\Omega^{\prime}, \Sigma^{\prime}, \mu^{\prime}\right)$ is the measure space of Theorem 3.1.

We extend $\phi_{1}: \mathcal{B} \rightarrow \mathcal{F}^{\prime}$ to simple functions, $\phi_{2}: \mathcal{S}\left(\mathcal{B}, \mu_{1}\right) \rightarrow T M\left(\Omega^{\prime}, \mathcal{F}^{\prime}, \mu^{\prime}\right):$

$$
\phi_{2}\left(\sum_{i=1}^{n} \alpha_{i} E_{i}\right)=\sum_{i=1}^{n} \alpha_{i} \phi_{1}\left(I_{E_{i}}\right) .
$$

Since $\mathcal{S}(\Omega, \mathcal{F}, \mu)$ is dense in $\dot{T} M(\Omega)$ we can extend $\phi_{2}$ continuously to an isomorphism

$$
\phi: \dot{T} M(\Omega, \mathcal{F}, \mu) \rightarrow \dot{T} M\left(\Omega^{\prime}, \Sigma^{\prime}, \mu^{\prime}\right)
$$

such that for all $\left\{f_{n}\right\} \in \dot{T} M(\Omega)$,

$$
\phi\left(\left\{f_{n}\right\}\right)=\lim _{n \rightarrow \infty} \phi_{2}\left(f_{n}\right)
$$

where the limit on the right hand side is the limit in the measure $\mu^{\prime}$. It is clear that $\phi\left(\left\{f_{n}\right\}\right) \in$ $\dot{T} M\left(\Omega^{\prime}, \Sigma^{\prime}, \mu^{\prime}\right)$. By the construction, $\left(\Omega^{\prime}, \Sigma^{\prime}, \mu^{\prime}\right)$ is a measure space and since in measure spaces, sequences which are Cauchy in measure converge in measure, we can identify the sequence $\left\{f_{n}\right\} \in$ $\dot{T} M\left(\Omega^{\prime}, \Sigma^{\prime}, \mu^{\prime}\right)$ with the limit in measure of $\left\{f_{n}\right\}$. In other words, $\dot{T} M\left(\Omega^{\prime}, \Sigma^{\prime}, \mu^{\prime}\right)=\mathcal{M}\left(\Omega^{\prime}, \Sigma^{\prime}, \mu^{\prime}\right)$, the space of measurable functions on $\left(\Omega^{\prime}, \Sigma^{\prime}, \mu^{\prime}\right)$.

From the definition of $\phi$ it is immediate that $\phi$ is an isometric isomorphism that preserves the order as defined in Definition 2.9, mapping $E \in \mathcal{B}$ to indicator functions $I_{\phi(E)} \in \mathcal{M}\left(\Omega^{\prime}, \Sigma^{\prime}, \mu^{\prime}\right)$.

The proofs of parts 2 and 3 in Theorem 3.1 may also be found in the original work, [4]. Closely following the proof of part 2 , we proceed to prove part 4 :

If $G \in \mathcal{B}$ then $G \in \dot{L}^{p, q}(\Omega, \mathcal{F}, \mu)$ and $\|G\|_{\dot{L}^{p, q}}^{q}=\left(\frac{p}{q} \mu_{1}(G)\right)^{1 / p}$. In this case $\phi^{-1}$ maps $\mu_{1}^{\prime}$-integrable simple functions in $L^{p, q}\left(\Omega^{\prime}, \Sigma^{\prime}, \mu^{\prime}\right)$ to elements in $\dot{L}^{p, q}(\Omega, \mathcal{F}, \mu)$ and preserves the $L^{p, q}$ norm. Therefore $\phi^{-1}$ takes $L^{p, q}\left(\Omega^{\prime}, \Sigma^{\prime}, \mu^{\prime}\right)$ into $\dot{L}^{p, q}(\Omega, \mathcal{F}, \mu)$ preserving norms. But $\dot{L}^{p, q}\left(\Omega^{\prime}, \mathcal{F}^{\prime}, \mu_{1}^{\prime}\right)=L^{p, q}\left(\Omega^{\prime}, \Sigma^{\prime}, \mu^{\prime}\right)$, so $\phi^{-1}$ takes $L^{p, q}\left(\Omega^{\prime}, \Sigma^{\prime}, \mu^{\prime}\right)$ isometrically into $\dot{L}^{p, q}(\Omega, \mathcal{F}, \mu)$.

If $E \in \mathcal{F}$ it is clear that $I_{\phi(E)} \in L^{p, q}\left(\Omega^{\prime}, \Sigma^{\prime}, \mu^{\prime}\right)$. Since $I_{E}=\phi^{-1}\left(I_{\phi(E)}\right)$, we have $I_{E} \in \operatorname{image}\left(\phi^{-1}\right)$. On the other hand, the linear span of $\left\{I_{E}: \mu(E)<\infty\right\}$ is dense in $\dot{L}^{p, q}(\Omega, \mathcal{F}, \mu)$. So $\phi^{-1}$ is onto. This completes the proof of the theorem.

Remark 3.9. By Theorem 3.1, any property of functions on a measure space that can be formulated in terms of operations preserved by the isomorphism $\phi,\|\cdot\|_{\dot{L}^{p}},\|\cdot\|_{\dot{L}^{p, q}}$, and the integral of $f$ is true for functions on a charge space. Note that one may prove many such statements without embedding a charge space into a measure space.

\section{Almost Periodic Functions}

\subsection{Shift invariant finitely additive probability charge on $\mathbb{R}$ and its extension to a mea- sure space.}

The following construction is commonly used in Ergodic Theory, see e.g. [8], pp. 39-41.

Consider the set function $\gamma$ on Lebesgue measurable subsets of $\mathbb{R}$ for which the following limit exists:

$$
\gamma(S)=\lim _{\tau \rightarrow \infty} \frac{\lambda(\{S \cap(-\tau, \tau)\})}{2 \tau} .
$$

In this section we extend $\gamma$ to a charge on $\mathcal{L}(\mathbb{R})$. 
Let $L^{\infty}(\mathbb{R}, \mathcal{L}, \lambda)$ be the space of $\lambda$-essentially bounded Lebesgue measurable functions with the norm $\|f\|_{L^{\infty}}=\lambda$-ess. $\sup _{t \in \mathbb{R}}|f(t)|$. Let

$$
\boldsymbol{M}=\left\{f \in L^{\infty}(\mathbb{R}):\left|\int_{a}^{b} f(s) d s\right| \leqslant B(f)<\infty \text { for all }-\infty<a \leqslant b<\infty\right\}
$$

Then $M$ is a subspace of $L^{\infty}(\mathbb{R}, \mathcal{L}, \lambda)$. Let $\tau_{h}(f)(x)=f(x+h)$ for all $h \in \mathbb{R}$.

For $f \in L^{\infty}(\mathbb{R}, \mathcal{L}, \lambda)$ let

$$
\boldsymbol{p}(\boldsymbol{f})=\lambda \text {-ess. } \sup _{t \in \mathbb{R}} f(t) .
$$

Then $p$ is a sublinear functional on $L^{\infty}(\mathbb{R}, \mathcal{L}, \lambda)$ that is nonnegative on $M$. Indeed if $p(f)=\lambda$-ess. $\sup _{t \in \mathbb{R}} f(t)=$ $\delta<0$ then $\left|\int_{-t}^{t} f(s) d s\right| \geqslant|\delta \cdot 2 t| \rightarrow \infty$ as $t \rightarrow \infty$ and therefore not bounded by any real number.

By the Hahn-Banach Theorem there exists a linear functional $T: L^{\infty}(\mathbb{R}, \mathcal{L}, \lambda) \rightarrow \mathbb{R}$, an extension of the 0 functional on $M$, bounded by the sublinear functional $p$.

$T$ satisfies the following properties.

1. $\boldsymbol{T}(\boldsymbol{f}) \geqslant 0 \forall f \geqslant 0$.

Since for $f \geqslant 0$,

$$
-T(f)=T(-f) \leqslant p(-f)=\lambda \text {-ess. } \sup _{t \in \mathbb{R}}(-f(t)) \leqslant 0 .
$$

2. $T(1)=1$.

Since

$$
T(1) \leqslant p(1)=1 \quad \text { and } \quad-T(1)=T(-1) \leqslant p(-1)=-1 .
$$

3. $T\left(\tau_{h}(\boldsymbol{f})\right)=\boldsymbol{T}(\boldsymbol{f})$ for all $h \in \mathbb{R}$.

Let $f \in L^{\infty}(\mathbb{R}, \mathcal{L}, \lambda),-\infty<a<b<\infty$ and $h \geqslant 0$. If $h \leqslant b-a$ then

$$
\begin{aligned}
\left|\int_{a}^{b}\left[f(s)-\tau_{h}(f)(s)\right] d s\right| & =\left|\int_{a}^{b}[f(s)-f(s+h)] d s\right|=\left|\int_{a}^{b} f(s) d s-\int_{a+h}^{b+h} f(s) d s\right| \\
& \leqslant\left|\int_{a}^{a+h} f(s) d s\right|+\left|\int_{b}^{b+h} f(s) d s\right| \leqslant 2 \cdot \lambda \text {-ess. } \sup _{t \in \mathbb{R}} f(t) \cdot h<\infty .
\end{aligned}
$$

If $h>b-a$ then

$$
\begin{aligned}
\left|\int_{a}^{b}\left[f(s)-\tau_{h}(f)(s)\right] d s\right| & =\left|\int_{a}^{b}[f(s)-f(s+h)] d s\right|=\left|\int_{a}^{b} f(s) d s-\int_{a+h}^{b+h} f(s) d s\right| \\
& \leqslant\left|\int_{a}^{b} f(s) d s\right|+\left|\int_{a+h}^{b+h} f(s) d s\right| \leqslant 2 \cdot \lambda \text {-ess. } \sup _{t \in \mathbb{R}} f(t) \cdot h<\infty
\end{aligned}
$$

proving that for all $h \geq 0,\left(f-\tau_{h}(f)\right) \in M$. Applying the result to $\tau_{-h}(f)$ for $h \geq 0$, we have $\left(\tau_{-h}(f)-f\right) \in M$ and so for all $h,\left(f-\tau_{h}(f)\right) \in M$. Since $T$ restricted to $M$ is zero,

$$
T(f)-T \tau_{h}(f)=T\left(f-\tau_{h}(f)\right)=0 .
$$

\section{4. $\lim _{|t| \rightarrow \infty}(\lambda$-ess.inf $f(t)) \leqslant T(f) \leqslant \lim _{|t| \rightarrow \infty}(\lambda$-ess.sup $f(t))$.}

From

$T(f) \leqslant p(f)=\lambda$-ess. $\sup _{t \in \mathbb{R}} f(t) \quad$ and $\quad-T(f)=T(-f) \leqslant p(-f)=\lambda$-ess. $\sup _{t \in \mathbb{R}}(-f(t))=-\lambda$-ess. $\inf _{t \in \mathbb{R}} f(t)$ 
it follows that

$$
\lambda \text {-ess. } \inf _{t \in \mathbb{R}} f(t) \leqslant T(f) \leqslant \lambda \text {-ess. } \sup _{t \in \mathbb{R}} f(t)
$$

If $T(f)>\lim _{|t| \rightarrow \infty} \lambda-$ ess. $\sup _{t \in \mathbb{R}} f(t)$ then $T(f)$ is larger than the essential supremum of $f$ on the interval $\left(-\infty,-t_{0}\right) \cup\left(t_{0}, \infty\right)$. But then

$$
T\left(f-f I_{\left[-t_{0}, t_{0}\right]}\right)=T(f)-T\left(f I_{\left[-t_{0}, t_{0}\right]}\right)=T(f)>\lambda-\text { ess. } \sup _{t \in \mathbb{R}}\left(f(t) I_{\left(-\infty,-t_{0}\right) \cup\left(t_{0}, \infty\right)}\right)
$$

which is a contradiction to (111)

5. $|T(f)| \leqslant\|f\|_{L^{\infty}}$.

Since

$$
T(f) \leqslant p(f)=\lambda \text {-ess. } \sup _{t \in \mathbb{R}} f(t) \leqslant \lambda \text {-ess. } \sup _{t \in \mathbb{R}}|f(t)|=\|f\|_{L^{\infty}}
$$

6. If $\lim _{|t| \rightarrow \infty} f(t)$ exists then $\boldsymbol{T}(\boldsymbol{f})=\lim _{|\boldsymbol{t}| \rightarrow \infty} \boldsymbol{f}(\boldsymbol{t})$.

This follows from property 4 .

Let $E$ be a Lebesgue measurable subset of $\mathbb{R}$ and

$$
\boldsymbol{\lambda}_{\boldsymbol{E}}(\boldsymbol{t})=\frac{\lambda(E \cap[-t, t])}{2 t} .
$$

We define

$$
\gamma(\boldsymbol{E})=T\left(\lambda_{E}\right)
$$

where $T$ is a Banach limit defined above.

To prove the additivity of $\gamma$ let $E, F \in \mathcal{L}(\mathbb{R})$ and $E \cap F=\varnothing$. Then

$$
\lambda_{E \cup F}(t)=\frac{\lambda((E \cup F) \cap[-t, t])}{2 t}=\frac{\lambda(E \cap[-t, t])}{2 t}+\frac{\lambda(F \cap[-t, t])}{2 t}=\lambda_{E}(t)+\lambda_{F}(t)
$$

Since $T$ is linear

$$
\gamma(E \cup F)=T\left(\lambda_{E \cup F}\right)=T\left(\lambda_{E}+\lambda_{F}\right)=\gamma(E)+\gamma(F)
$$

By Property 6 of $T$, when the following limit exists, we have

$$
\gamma(E)=\lim _{t \rightarrow \infty} \frac{\lambda(E \cap[-t, t])}{2 t}
$$

Since

$$
\gamma(\varnothing)=\lim _{t \rightarrow \infty} \frac{\lambda(\varnothing \cap[-t, t])}{2 t}=0
$$

and

$$
\gamma(\mathbb{R})=\lim _{t \rightarrow \infty} \frac{\lambda(\mathbb{R} \cap[-t, t])}{2 t}=1,
$$

$(\mathbb{R}, \mathcal{L}(\mathbb{R}), \gamma)$ is a probability charge space.

Definition 4.1. We say that $E \subseteq \mathbb{R}$ is a $q$-periodic set if $I_{E}$ is a $q$-periodic function, that is, periodic with period $q$.

Theorem 4.2. For any q-periodic measurable set $E \subseteq \mathbb{R}$ and any $a \in \mathbb{R}$,

$$
\gamma(E)=\lim _{t \rightarrow \infty} \frac{\lambda(E \cap[-t, t])}{2 t}=\frac{\lambda(E \cap[a, a+q])}{q}
$$


Corollary 4.3. If $f$ is a $q$-periodic function on $\mathbb{R}$, then for any $s>0, E_{s}=\{|f|>s\}$ is a $q$-periodic set, and therefore for any $a \in \mathbb{R}$

$$
\gamma_{f}(s)=\lim _{t \rightarrow \infty} \frac{\lambda(\{|f|>s\} \cap[-t, t])}{2 t}=\frac{\lambda(\{|f|>s\} \cap[a, a+q])}{q} .
$$

Theorem 4.4. If $f$ is an integrable q-periodic function, then for any $a \in \mathbb{R}, \int_{\mathbb{R}} f d \gamma=\frac{1}{2 q} \int_{a-q}^{a+q} f(x) d x$ Proof. Let $f$ be a non-negative integrable $q$-periodic function. Since $f$ is periodic,

$$
\gamma_{f}(s)=\lim _{t \rightarrow \infty} \frac{\lambda(\{f>s\} \cap[-t, t])}{2 t}=\frac{\lambda(\{f>s\} \cap[-q, q])}{2 q}=\frac{1}{2 q} \lambda_{f I_{[-q, q]}}(s) .
$$

By Lemma (2.21),

$$
\int_{\mathbb{R}} f d \gamma=\int_{0}^{\infty} \gamma_{f}(s) d s=\frac{1}{2 q} \int_{0}^{\infty} \lambda_{f I_{[-q, q]}}(s) d s=\frac{1}{2 q} \int_{-q}^{q} f(x) d x=\frac{1}{2 q} \int_{a-q}^{a+q} f(x) d x
$$

proving the claim for non-negative functions. More generally, if $f$ is any $q$-periodic integrable function, we apply (122) to $f^{+}$and to $f^{-}$.

Theorem 4.5. Any almost periodic trigonometric polynomial $P_{n}(x)$ is integrable over $(\mathbb{R}, \mathcal{L}(\mathbb{R}), \gamma)$ with

$$
\int_{\mathbb{R}} P_{n} d \gamma=\lim _{T \rightarrow \infty} \frac{1}{2 T} \int_{-T}^{T} P_{n}(x) d x
$$

Proof. Apply Theorem 4.4 to each of the terms, $e^{i \eta_{k} x}$.

Applying (122) to (5),

Theorem 4.6. $\left\{e^{i \eta_{k} x}\right\}$ with $\eta_{k} \in \mathbb{R}, k \in \mathbb{N}$ is an orthonormal system in $L^{2}(\mathbb{R}, \mathcal{L}(\mathbb{R}), \gamma)$.

Corollary 4.7 (Bessel's Inequality). If $|f|^{2}$ is integrable over $(\mathbb{R}, \mathcal{L}(\mathbb{R}), \gamma)$ and $\eta_{k}$ are all different, then

$$
\sum_{k=0}^{\infty}\left|\int_{\mathbb{R}} f(x) e^{-i \eta_{k} x} d \gamma\right|^{2} \leq \int_{\mathbb{R}}|f|^{2} d \gamma
$$

\section{A Paley type theorem for almost periodic functions}

We define the following extension of the Besicovitch $B_{a p}^{p}$ space.

Definition 5.1. For $0<p, q<\infty$ define $B_{a p}^{p, q}(\mathbb{R}, \mathcal{L}(\mathbb{R}), \gamma)$ to be the completion of almost periodic

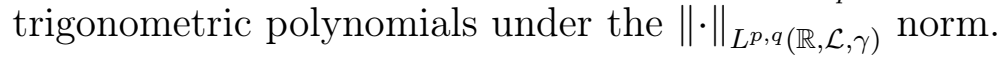

By Remark $3.9, L^{p, p}(\mathbb{R}, \mathcal{L}(\mathbb{R}), \gamma)=L^{p}(\mathbb{R}, \mathcal{L}(\mathbb{R}), \gamma)$, and so the theory of the spaces $B_{a p}^{p, q}(\mathbb{R}, \mathcal{L}(\mathbb{R}), \gamma)$ is an extension of the theory of the Besicovitch spaces $B_{a p}^{p}$.

We also have the following result.

Theorem 5.2. If $f \in L^{1}(\mathbb{R}, \mathcal{L}(\mathbb{R}), \gamma)$ then

$$
\left|a\left(\eta_{k}, f\right)\right|=\left|\int_{\mathbb{R}} f(t) e^{-i \eta_{k} t} d \gamma(t)\right| \leqslant\|f\|_{L^{1}(\mathbb{R}, \mathcal{L}(\mathbb{R}), \gamma)}\left\|e^{-i \eta_{k} t}\right\|_{L^{\infty}} \leqslant\|f\|_{L^{1}(\mathbb{R}, \mathcal{L}(\mathbb{R}), \gamma)}
$$


Let $\boldsymbol{\eta}$ be the counting measure on $2^{\mathbb{R}}$. Then the generalized Fourier operator is linear and maps $L^{1}(\mathbb{R}, \mathcal{L}(\mathbb{R}), \gamma)$ into $L^{\infty}\left(\mathbb{R}, 2^{\mathbb{R}}, \eta\right)$ and $L^{2}(\mathbb{R}, \mathcal{L}(\mathbb{R}), \gamma)$ into $L^{2}\left(\mathbb{R}, 2^{\mathbb{R}}, \eta\right)$ continuously.

Theorem 5.3. Let $1<p<2$ and $0<q<\infty, 1 / p+1 / p^{\prime}=1$. If $f \in L^{p, q}(\mathbb{R}, \mathcal{L}(\mathbb{R}), \gamma)$, with

$$
a\left(\eta_{k}, f\right)=\int_{\mathbb{R}} f(t) e^{-i \eta_{k} t} d \gamma(t), \quad \eta_{k} \in \mathbb{R} .
$$

Then $\left\{a\left(\eta_{k}, f\right)\right\}_{k \in \mathbb{N}} \in L^{p^{\prime}, q}\left(\mathbb{R}, 2^{\mathbb{R}}, \eta\right)$ and

$$
\left\|\left\{a\left(\eta_{k}, f\right)\right\}\right\|_{L^{p^{\prime}, q\left(\mathbb{R}, 2^{\mathbb{R}}, \eta\right)}} \leqslant C(p, q)\|f\|_{L^{p, q}(\mathbb{R}, \mathcal{L}(\mathbb{R}), \gamma)} .
$$

Proof. By Theorem 3.1 part (4) the spaces $L^{1}(\mathbb{R}, \mathcal{L}(\mathbb{R}), \gamma), L^{\infty}\left(\mathbb{R}, 2^{\mathbb{R}}, \eta\right), L^{2}(\mathbb{R}, \mathcal{L}(\mathbb{R}), \gamma)$, and $L^{2}\left(\mathbb{R}, 2^{\mathbb{R}}, \eta\right)$ are isometrically isomorphic to $L^{1}\left(\mathbb{R}^{\prime}, \mathcal{L}^{\prime}(\mathbb{R}), \gamma^{\prime}\right), L^{\infty}\left(\mathbb{R}^{\prime}, 2^{\mathbb{R}^{\prime}}, \eta^{\prime}\right), L^{2}\left(\mathbb{R}^{\prime}, \mathcal{L}^{\prime}\left(\mathbb{R}^{\prime}\right), \gamma^{\prime}\right)$, and $L^{2}\left(\mathbb{R}^{\prime}, 2^{\mathbb{R}^{\prime}}, \eta^{\prime}\right)$ so (124) and (125) holds for the measure spaces. By Marcinkiewicz-Hunt, [6], ([5] p. 52), (127) holds for the corresponding measure spaces and therefore (127) follows.

Remark 5.4. One may similarly prove the Marcinkiewicz-Hunt theorem for finitely additive measure spaces for $0<p_{i}, q_{i}<\infty$. 


\section{References}

[1] A. Avantaggiati, G. Bruno, and R. Iannacci. The Hausdorff-Young theorem for Almost Periodic Functions and Some Applications. Nonlinear Analysis, 25(1):61-87, 1995.

[2] Y. Boryshchak. Norm Inequalities for the Fourier Coefficients of Some Almost Periodic Functions. Doctoral dissertation, 2019.

[3] N. Dunford and J. T. Schwartz. Linear Operators Part I: General Theory, volume 7. Interscience Publishers New York, 1958.

[4] C. Fefferman. $L_{p}$ Spaces over Finitely Additive Measures. Pacific Journal of Mathematics, 26(2):265-271, 1968.

[5] L. Grafakos. Classical Fourier Analysis, volume 2. Springer, 2008.

[6] R. Hunt. On L $(p, q)$ Spaces, l'Enseignement Math. 12 (1966), 249-276. Google Scholar.

[7] R. Paley. A Proof of a Theorem on Bilinear Forms. Journal of the London Mathematical Society, 1(3):226-230, 1931.

[8] K. B. Rao and M. B. Rao. Theory of Charges: A Study of Finitely Additive Measures, volume 109. Academic Press, 1983.

[9] J. Yeh. Real Analysis: Theory of Measure and Integration Second Edition. World Scientific Publishing Company, 2006.

[10] A. Zygmund. Trigonometric Series, volume 1. Cambridge University Press, 2002. 